\title{
Locating a small change in a multiple scattering environment
}

Eric Larose, ${ }^{1}$. 2 Thomas Planes, ${ }^{1}$ Vincent Rossetto, ${ }^{2}$ and Ludovic Margerin ${ }^{3}$

1) Lab. de Géophysique Interne et Tectonophysique, CNRS \& Univ. J. Fourier, BP53, 38041 Grenoble, France.

${ }^{2)}$ Lab. de Physique et Modélisation des Milieux Condensés,

CNRS 8 Univ. J. Fourier, BP 166, 38042 Grenoble, France.

3) Centre Européen de Recherche et d'Enseignement des Géosciences de

l'Environnement, CNRS \& Univ. Aix Marseille Europôle Méditerranéen de l'Arbois, BP 80, 13545 Aix en Provence, France

(Dated: 19 July 2010)

This article presents an imaging technique to locate a weak perturbation in a multiple scattering environment. We derive a formula to predict the spatio-temporal decorrelation of diffuse coda waves induced by an extra-scatterer. Locating this new defect is formulated as an inverse problem which is solved by a maximum likelihood approach. Using elastic waves in the $50-400 \mathrm{kHz}$ frequency band, we recover the position of a millimetric hole drilled in a concrete sample with a precision of a few $\mathrm{cm}$. Note that the size of the defect is comparable to the size of the myriads of heterogeneities constituting the sample.

PACS numbers: 43.40.+s, 43.20.+g, 46.40.Cd, 91.30.-f

\footnotetext{
a) Electronic mail: eric.larose@ujf-grenoble.fr
} 
Waves constitute the primary tool to detect and locate temporal changes in natural or man-made structures. Numerous important applications can be found in every day life, including ultrasonic medical imaging, non-destructive testing, seismic exploration, radar aircraft location, sonar... The most common techniques rely on the emission of a controlled pulse (of central period $T_{0}$ ) into the medium, which is back-scattered by one or several targets, and recorded at one or several receivers. These pulse-echo approaches are based on the single scattering assumption and are operated in weakly heterogeneous media where the typical propagation time $T_{R}$ is much smaller than the scattering mean-free time $\tau$. In a heterogeneous multiple-scattering medium, it is well understood that the waves lose memory of their initial direction of propagation beyond a time scale known as the transport mean free time $\tau^{\star}$. This feature hampers the location or detection of new targets in a slowlyevolving medium (with time scale $d$ ), based on conventional pulse-echo techniques. When the following relation applies:

$$
T_{0} \ll \tau^{\star} \ll T_{R} \ll d,
$$

wave propagation is similar to a random walk and can be described by a diffusion equation. The mesoscopic regime (11) prevails in many solid materials including iron polycrystals, concrete or multicomposite materials, for which non-destructive evaluation is required.

The problem of locating an isolated change in a multiple scattering sample has received some attention in the past. In optics, the most powerful techniques are based on the variation of intensity in a speckle pattern probed by one or more receivers] 1 . In the case of collective changes in the medium, the diffusive wave spectroscopy and its variants have become standard tools. In the case of local changes, previous authors defect -the perturbation- added to a collection of scatterers -the background- essentially acts as a dipole source of intensity. This has been confirmed by the numerical studyl. These authors also find that the method has weak sensitivity and that a large amount of ensemble or frequency averaging (typically 100 realizations) is required to distinguish the intensity fluctuation caused by the defect from the background speckle pattern. This renders techniques based on intensity variations almost inapplicable to solid media. Other authors proposed to interpret the changes of waveforms in the coda in terms of traveltime variations or phase differences 6 . This approach was found to be very sensitive to detect small changes, but gives little information on the location of the change. Recently, Aubry and Derodel proposed an alternative technique based on the singular value decomposition of the propagator, 
but this technique is limited to a strong extra scatterer and is not sensitive to weak perturbations. In this article, we report on a different approach to locate a small isolated change. Our LOCADIFF technique uses simultaneously the information carried by the phase and the amplitude of a wavefield. The imaging technique results in a three-dimensional (3D) map representing the likelihood of the change location.

The decorrelation of coda waves induced by the addition of a small defect is best illustrated by a laboratory experiment. We consider a block of concrete which is a common composite and heterogeneous material. The average elastic wave speeds in the sample are $\approx 4300 \mathrm{~m} / \mathrm{s}$, and $\approx 2500 \mathrm{~m} / \mathrm{s}$ for compressional and shear waves, respectively. The corresponding wavelengths range from a few millimeters to a few centimeters in the $50-400 \mathrm{kHz}$ frequency range. The $1 \mathrm{~m} \times 1 \mathrm{~m} \times 0.25 \mathrm{~m}$ block consists in a mixture of cement, millimetric sand and centimetric gravel. These heterogeneities render the medium multiply scattering for ultrasound above $100 \mathrm{kH}$. Height ultrasonic transducers $R$ are glued onto the sample (see Fig. 1), either operated as sources or receivers. The experiment consists in successive acquisitions of the impulse responses $h_{i j}^{d}(t)$ for all available pairs of transducers at various dates $d$. Subscript $i$ (resp. $j$ ) holds for transducer $i$ (resp. $j$ ) located at $\mathbf{r}_{i}$ (resp. $\mathbf{r}_{j}$ ).

In Fig. 1 (bottom) we plot an example of an ultrasonic record between $R_{0}$ and $R_{3}$. The broken gray line shows the theoretical envelope $\sqrt{g_{i j}(t)}$ obtained from solutions of the 3D diffusion equation for a point source at $\mathbf{r}_{\mathbf{0}}$ and detection at $\mathbf{r}$ :

$$
\left.\partial_{t} g\left(\mathbf{r}, \mathbf{r}_{0}\right)+\frac{1}{\tau_{a}} g\left(\mathbf{r}, \mathbf{r}_{0}\right)-D \Delta_{r} g\left(\mathbf{r}, \mathbf{r}_{0}\right)\right)=\delta\left(\mathbf{r}-\mathbf{r}_{0}\right) \delta(t)
$$

where $g$ also includes reflections from the sides. The diffusion constant $D=25 \mathrm{~mm}^{2} / \mu \mathrm{s}$ and the absorption time $\tau_{a}=200 \mu \mathrm{s}$ are derived from ref. 10 and are found to properly fit our experimental data.

In order to detect (and, subsequently, locate) an isolated change, we compare the coda waveforms obtained at different dates. In the first experiment, four sets of impulse responses $h_{i j}^{d}$, where $d=0 . .3$, are acquired over the first 60 minutes of the experiment; then a small hole (3 $\mathrm{mm}$ in diameter and $12 \mathrm{~mm}$ in height) is drilled on the top of the block, and four additional sets of records $(d=4 . .7)$ are acquired over another 80 minutes. In the second experiment, we reproduce the same protocole but the hole is $8 \mathrm{~mm} \times 22 \mathrm{~mm}$ and drilled on the other side of the block. For each record $h_{i j}^{d}$, and at each date $d$, we evaluate the 
decorrelation coefficient $K_{i j}^{d}(t)$ as follows:

$$
K_{i j}^{d}(t)=1-\frac{\int h_{i j}^{0}(u) h_{i j}^{d}(u) d u}{\sqrt{\int h_{i j}^{0}(u)^{2} d u \int h_{i j}^{d}(u)^{2} d u}}
$$

where the integration is performed from 500 to $1500 \mu \mathrm{s}$. This experimental decorrelation $K_{i j}^{d}$ is plotted in Fig. 目 versus the time in the experiment for three pairs of transducers. We observe that coda waves have decorrelated right after the hole was drilled. This feature is not new and forms the basis of original detection and characterization techniquest. Going from detection to location requires additional information: we will take into account the spatial dependence of the decorrelation (see Fig. 2). From previous theoretical works on multiple scattering, we can derive a theoretical prediction for the decorrelation due to an extra-scatterer in $\mathbf{r}$ :

$$
K_{i j}^{n}(\mathbf{r}, t)=\frac{c \sigma \int_{0}^{t} g\left(\mathbf{r}_{j}, \mathbf{r}, t-u\right) g\left(\mathbf{r}, \mathbf{r}_{i}, u\right) d u}{g\left(\mathbf{r}_{i}, \mathbf{r}_{j}, t\right)}
$$

where $\sigma$ denotes the extinction cross-section of the defect, and where only scalar waves are considered. Equation (4) is the theoretical model that forms the basis of our inversion procedure. $(c \sigma)^{-1} K_{i j}^{n}(\mathbf{x}, t) d^{3} \mathbf{x}$ can be interpreted as the probability that a random walk starting at $\mathbf{r}_{i}$ and ending at $\mathbf{r}_{j}$ after a time $t$ passes at least once in the small volume $d^{3} \mathbf{x}$ surrounding $\mathbf{x}$. In our sample, we can approximate the propagator $g$ with a causal version of the diffusion equation. This approximation also avoids divergence of the integrand at short and large $u$ in equation (4).

Finding the location $\mathbf{r}$ of the defect from the set of experimental $K_{i j}^{d}$ defines our inverse problem. To solve this inverse problem, we discretize the medium into a regular 3-D grid. Each point $\mathbf{x}$ of the grid is considered as a candidate location for the apparition of the defect: for each point $\mathbf{x}$ and each couple of receivers $\{i, j\}$ a numerical decorrelation $K_{i j}^{n}(\mathbf{x})$ is evaluated after eq. (4). Following the maximum-likelihood approach 14 , we calculate the relative residual error $\chi^{2}(\mathbf{x})$, also called the misfit function, between experimental $K^{d}$ and numerical $K^{n}$ data:

$$
\chi^{2}(\mathbf{x})=\sum_{i, j}\left(K_{i j}^{d}(t)-K_{i j}^{n}(\mathbf{x}, t)\right)^{2} / \epsilon^{2} .
$$

The parameter $\epsilon$ accounts for measurement errors and uncertainties in the transport parameters. Using measurements of the decorrelation in different time windows around 
time $t$, we inferred a typical standard-deviation $\epsilon=0.6 \%$. Since the scattering cross-section of the defect is unknown, we also explored values of $c \sigma$ ranging from 0.1 to $20 \mathrm{~cm}^{3} / \mu \mathrm{s}$ and selected the optimal results based on $\chi^{2}$ minimization and stability with respect to small changes in $\sigma$. The result of the inversion procedure is the relative residual error (misfit function) normalized by the number of degree of freedom $\chi_{n}^{2}$. In Fig. 3 we show a slice of $\chi_{n}^{2}$ at the top side of the sample $(\mathrm{z}=25 \mathrm{~cm})$ for exp. $\# 1$, and at the bottom side $(\mathrm{z}=0 \mathrm{~cm})$ for exp. \#2. The crosses indicating the location of the defects coincides remarkably well with the minimum of the misfit function.

The crucial physical parameter in our inversion is the lapse time $t$ in the coda for which the decorrelation is evaluated. In the late coda, the sensitivity to the defect is maximum, but the decorrelation depends only algebraically on the defect location and the decorrelation tends to spatially homogenize on the scale of our sample. On the other hand, the decorrelation at early times shows rapid spatial variations but its absolute value is very small and within the experimental error. Therefore, there exists a trade-off between lateral resolution and sensitivity which points towards the existence of an optimal lapse time, for which the best compromise is obtained 15 .

In this article, we have presented an imaging technique, named LOCADIF, to locate a small change in a multiple scattering medium. Our technique has the following prerequisites: 1) estimate of the transport properties; 2) reproducible experimental apparatus; 3) calculation of the transport intensity between any point in the probed medium; 4) geometry of the medium. The technique was proved to efficiently detect and locate a small hole in two different experimental configurations. In the first experiment, a $3 \mathrm{~mm} \times 12 \mathrm{~mm}$ is drilled on the same side than the transducers. In the second experiment, a $8 \mathrm{~mm} \times 22 \mathrm{~mm}$ hole is drilled on the other side. In both cases, the position of the defect is retrieved in 3D with a resolution of a few $\mathrm{cm}$ (including the vertical direction). Note that the size of the hole is comparable to the size of the heterogeneities constituting the sample, and that such a defect is hardly detected by conventional ultrasonic techniques because multiple scattering hampers the de-

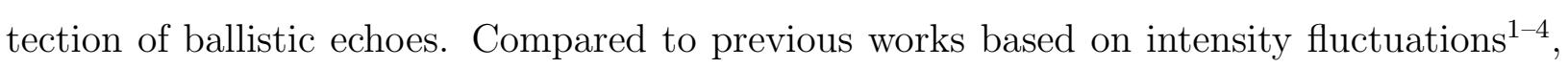
our method has much higher sensitivity and take advantage of broadband waveforms. The technique is applicable to any kind of classical waves propagating in highly heterogeneous media, provided that those waves are probed both in phase and amplitude. We suggest ap- 
plications to non-destructive testing and monitoring of multi-composite materials, including civil engineering structures 16 . Applications to monitoring dynamic geophysical media are also on the way.

We acknowledge help from N. Tremblay. This work was funded by the ANR JC08_313906 SISDIF grant. 


\section{REFERENCES}

${ }^{1}$ D. J. Pine, D. A. Weitz, P. M. Chaikin, and E. Herbolzheimer, Phys. Rev. Lett. 60, 1134 (1988).

${ }^{2}$ S. Feng and D. Sornette, J. Acoust. Soc. Am. 90, 1742 (1991).

${ }^{3}$ C. Vanneste, S. Feng, and D. Sornette, europhys. lett. 24, 339 (1993).

${ }^{4}$ M. C. W. van Rossum and T. M. Nieuwenhuizen, Rev. Mod. Phys. 71, 313 (1999).

${ }^{5}$ T. M. Nieuwenhuizen and M. C. W. van Rossum, Physics Letters A 177, 102 (1993).

${ }^{6}$ M. L. Cowan, J. H. Page, and D. A. Weitz, Phys. Rev. Lett. 85, 453 (2000).

${ }^{7}$ O. I. Lobkis and R. L. Weaver, Phys. Rev. Lett. 90, 254302 (2003).

${ }^{8}$ C. Pacheco and R. Snieder, J. Acoust. Soc. Am. 118, 1300 (2005).

${ }^{9}$ A. Aubry and A. Derode, Phys. Rev. Lett. 102, 084301 (2009).

${ }^{10}$ P. Anugonda, J. S. Wiehn, and J. A. Turner, ultrasonics 39, 429 (2001).

${ }^{11}$ R. Snieder, A. Grêt, H. Douma, and J. Scales, Science 295, 2253 (2002).

${ }^{12}$ J. De Rosny and P. Roux, J. Acoust. Soc. Am. 109, 2587 (2001).

${ }^{13}$ J. E. Michaels and T. E. Michaels, IEEE trans. Ultrason., Ferroelect., Freq. Contr. 52, 1769 (2005).

${ }^{14}$ W. H. Press, S. A. Teukolsky, W. T. Vetterling, and B. P. Flannery, Numerical Recipes in $C$ (Cambridge University Press, Cambridge, 1992).

${ }^{15}$ See supplementary material at.

${ }^{16}$ Patent number FR09-50612. 

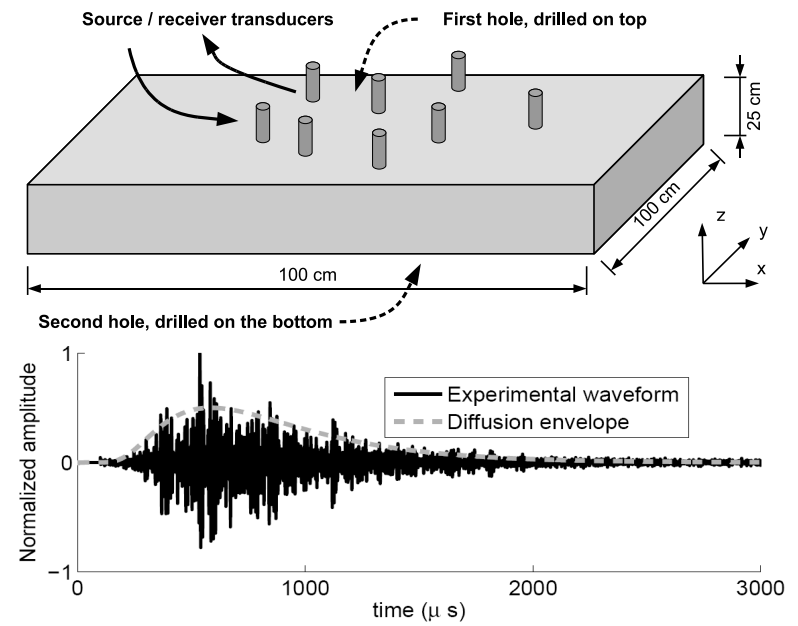

FIG. 1. Setup for experiment \#1\&2 (top) and typical ultrasonic waveform (bottom). The concrete block is equipped with height piezoelectric transducers. After starting the experiment, a hole is drilled on the top side (exp. \#1) or on the bottom side (exp. \#2) of the block.

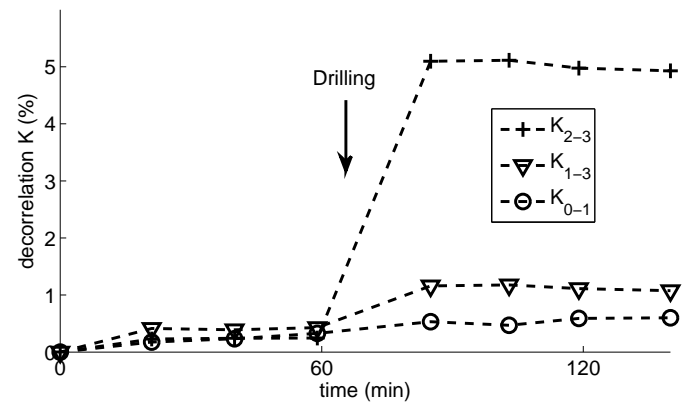

FIG. 2. Decorrelation $K^{d}$ for transducers $0-1,1-3$ and 2-3 evaluated between 500 and $1500 \mu s$. The hole drilled after $60 \mathrm{~min}$ is perfectly detected in the coda. The decorrelation strongly depends on the position of the transducers with respect to the change. 

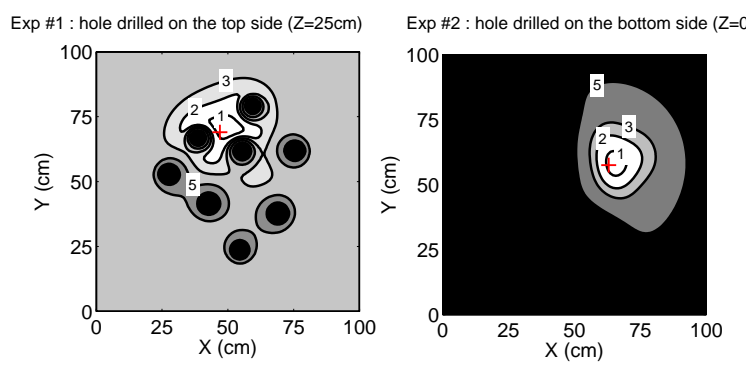

FIG. 3. Slices of the normalized residual error $\chi_{n}^{2}$ after the inversion. Left: on the top side $(\mathrm{z}=25 \mathrm{~cm})$ during experiment \#1. Right: at the bottom side $(\mathrm{z}=0 \mathrm{~cm})$ during experiment $\# 2$. The defect positions are highly likely within the white areas defined by $\chi_{n}^{2}<1$. The actual position of the holes are marked with a cross. The black areas where $\chi_{n}^{2}>10$ mark the positions where the defect is very unlikely. Note that for exp. \#1, these black areas also correspond to the positions of the transducers. 